\title{
THE SALVO COMBAT MODEL WITH AREA FIRE ${ }^{1}$
}

\author{
Michael J Armstrong \\ Goodman School of Business, Brock University \\ St Catharines, ON, L2S 3A1, Canada \\ michael.armstrong@brocku.ca
}

\begin{abstract}
This paper analyzes versions of the salvo model of missile combat where area fire is used by one or both sides in a battle. While these models share some properties with the area fire Lanchester model and the aimed fire salvo model, they also display some interesting differences, especially over the course of several salvos. Whereas the relative size of each force is important with aimed fire, with area fire it is the absolute size that matters. Similarly, while aimed fire exhibits square law behavior, area fire shows approximately linear behavior. When one side uses area and the other uses aimed fire, the model displays a mix of square and linear law behavior.
\end{abstract}

Keywords: military; missile combat; salvo equations; Lanchester equations.

\section{INTRODUCTION}

In battle it is desirable to have precise information about enemy dispositions. For example, consider a naval task force that encounters hostile warships in mid-ocean. Each side's radar would presumably provide good information about enemy positions and allow accurate aiming of anti-shipping cruise missiles (ASCMs). This aimed fire scenario could appropriately be represented by the salvo model of missile combat developed by Hughes [7].

However, navies do not always have such precise information. Suppose the task force next approaches the enemy coastline and comes into range of their land-based defenses (e.g., truck-mounted ASCMs), as in [11]. If those land forces are concealed by camouflage, then the naval force might need to attack while knowing only their approximate positions. Conversely, the land forces might be able to attack using radar to pinpoint the exact location of the naval force; or they might instead need to rely on signals intelligence to estimate the navy's approximate location amid the littoral clutter of islands, fishing boats, etc.

An attack against targets whose locations are known only approximately is sometimes referred to as area fire. In the context of artillery guns exchanging counter-battery fire, this can be modeled by the area fire version of the Lanchester equations [12]. Those equations also serve as one-half of the Lanchester ambush fire model, in which one side uses aimed fire while the other side uses area fire. This hybrid model can represent firefights in counter-insurgency operations, where the insurgents conceal themselves within overgrown terrain [4] or urban populations [8]. The insurgents use aimed fire when shooting at regular army troops, and the regulars shoot back using area fire.

\footnotetext{
${ }^{1}$ This is the peer reviewed version of the following article: [Armstrong MJ, 2013, "The salvo combat model with area fire", Naval Research Logistics 60 \#8, 652-660], which has been published in final form at http://onlinelibrary.wiley.com/doi/10.1002/nav.21559/abstract. This article may be used for non-commercial purposes in accordance with Wiley Terms and Conditions for Self-Archiving.
} 
Area fire was first applied to salvo combat by Mahon [11] in a thesis supervised by Hughes and Lucas. Mahon used a hybrid model to study littoral combat between naval and land forces. He assumed that the land forces (truck-mounted missile launchers) had little staying power and no active defenses, but were partially concealed; therefore the naval force would use area fire when attacking them. Meanwhile, the naval force was protected by active defenses but exposed to aimed fire from the land-based ASCMs. Mahon's numerical studies suggested that area fire would put the navy at a serious disadvantage.

Area fire could also arise where one side has a significant technological advantage over the other, as with, e.g., a United States Navy (USN) task force engaging a smaller country's coastal patrol boats. The USN could use its phased-array radars, drones, and aircraft to precisely locate its opponents at long range; its attack would therefore be modeled as aimed fire. By contrast, the opposing patrol boats have only basic ship-board radars and must close the range in order to pinpoint the USN's location. However, they would likely be sunk by the USN before it gets close enough to do that. The patrol boats therefore might reasonably attack while knowing only the approximate location of the USN (e.g., by firing a spread of ASCMs in their general direction), because that would be better than sinking without firing a shot.

The use of counter-targeting measures [9] such as chaff, jamming, and decoys by one or both sides to obscure their locations could lead to area fire as well. Several contacts may appear on a radar screen, but only some are valid targets; the rest are chaff clouds or active decoys. Or the extra contacts might be vessels that do not interest the attacker, such as warships crippled by a previous salvo but still afloat. The attacker might prefer to fire at all of the contacts, rather than risking a delay to sort out which ones matter. Weapons that carry sub-munitions to blanket the area would be useful here, as these are inherently area-effect in nature. (There is speculation that the new DF-21 anti-ship ballistic missile has this capability [14]).

This paper builds on Mahon's numerical work by performing an analytical study of salvo combat models using area fire. It first considers the case where both sides use area fire. This "pure" area fire case is less likely to arise in actual combat, but it makes the definition (Section 3 ) and analysis (Section 4) of area fire salvos relatively straightforward.

The analysis uses an approach similar to [3], and finds that the area fire salvo model has some approximately linear law properties similar to its Lanchester equivalent. Thus a quantitative advantage held by one side can be countered with a comparable qualitative advantage by the other. However, the area fire salvo model displays a wider range of battle outcomes than does the Lanchester model. For example, conditions can exist in the area fire salvo model where the initial salvo exchange causes losses to both sides, but subsequent salvos cause no damage.

Section 5 studies the case where one side uses area fire while the other uses aimed fire. This asymmetric or hybrid salvo model displays a combination of square and linear law behavior. The side using aimed fire has a significant advantage that can compensate for qualitative or quantitative inferiority; however, that advantage shrinks as force sizes increase. 
Section 6 shows how a force using area fire could improve its effectiveness by concentrating its firepower on a subset of the target area. Section 7 concludes by discussing the models' implications and limitations. Appendix 1 contains the details of all mathematical derivations, and Appendix 2 provides a numerical example to illustrate model calculations.

\section{THE AIMED FIRE SALVO MODEL}

This section briefly reviews the original aimed fire salvo combat model [7]. Consider a battle between two forces, Red and Blue. Let $A$ represent the number of combat units (warships or other weapon platforms) in the Red force at the beginning of the battle. Each one has offensive firepower $\alpha$, which is the number of offensive missiles accurately fired per salvo at the enemy. Each one also has defensive firepower $y$, which is the number of incoming enemy missiles intercepted per salvo by active defenses. Each missile that is not intercepted causes the loss of a fraction $u$ of a Red unit, so that the staying power $w=1 / u$ is the number of hits needed to put one unit out of action. Similar symbols represent Blue's offensive firepower $\beta$, defensive firepower $z$, loss per hit $v$, and staying power $x=1 / v$, as shown in Table 1 .

The battle begins with Red firing a salvo of missiles at Blue, who tries to intercept the incoming missiles. Simultaneously, Blue launches a salvo that Red tries to intercept. The salvo equations calculate the changes in strength $\Delta B$ for Blue and $\Delta A$ for Red as follows [7].

$$
\begin{array}{lll}
\Delta B=-(\alpha A-z B) v & \text { subject to } & 0 \leq-\Delta B \leq B \\
\Delta A=-(\beta B-y A) u & \text { subject to } & 0 \leq-\Delta A \leq A
\end{array}
$$

\section{INCORPORATING AREA FIRE}

To adapt the salvo model for area fire, some measure of the relative size of the area being attacked must be incorporated. For this purpose, Mahon [11] defined a parameter called the munitions lethal targeted area ratio that compares the area affected by each weapon round to the total area in which the enemy is located. It reflects how precisely the target locations are known, relative to the characteristics of the weapon used. Herein the symbol $0<m<1$ represents the targeted area ratio when Red is attacking, and $0<n<1$ when Blue is attacking. These ratios for the attackers' weapons are then multiplied by the numbers of the defenders' units, as in $m B$ when Red is firing and $n A$ when Blue is firing, to create a measure of target density (e.g., ships per square kilometer). Thus $m B$ and $n A$ indicate the proportions, between 0 and 1 , of missiles that are headed towards actual targets rather than empty space. One may think of this as the extent to which offensive firepower is diluted before reaching defending units.

The area fire studied in [11] mostly involved naval gunfire aimed at land-based targets, so the targeted area ratio modeled the lethal area of each shell blast relative to the total land area within which the targets were located. The numerical examples in that work used $m$ in the range of 0.001 to 0.01 , and target densities $m B$ of around 0.004 to 0.08 . For ASCMs fired at warships using counter-targeting measures, $m$ could represent the swept area of the missile's seeker, relative to the total area containing the ships. In this context, higher values of $m$ and $m B$ might be relevant; e.g., if 1 warship deploys 3 radar decoys, then $m B=0.25$. 
To better understand this concept, imagine the target area being divided into small subsections. Each subsection corresponds to the area within which a target would be affected by a weapon round. When Red shoots at Blue, Red knows that there are $B$ Blue targets spread across $1 / m$ subsections. Proportion $B /(1 / m)=m B$ of the subsections actually contains targets, while proportion $(1-m B)$ does not. Red spreads its attacking salvo $\alpha A$ evenly across all the subsections; Blue intercepts only those missiles that are heading for occupied subsections.

This leads to the following equations for the area fire salvo combat model. (See Appendix 2 for example calculations with these and subsequent equations.)

$$
\begin{array}{lll}
\Delta B=-(\alpha A m B-z B) v & \text { subject to } & 0 \leq-\Delta B \leq B \\
\Delta A=-(\beta B n A-y A) u & \text { subject to } & 0 \leq-\Delta A \leq A
\end{array}
$$

Note that the model assumes $A<1 / n$ and $B<1 / m$. Larger forces than these would completely fill the target area, in which case the analysis should switch to the aimed fire model.

As with the Lanchester area fire model, this formulation assumes that the defending units are spread evenly across a common area of fixed dimensions; e.g., all the defenders are hiding somewhere on a given map. If more targets are added, then more subsections of that map become occupied and the target density increases. This is sometimes called the constant area case [12]. In other contexts, however, each defending unit might come with its own area; e.g., when more defenders are added, the map gets larger. This is called the constant density case because adding more targets increases their number but also the total area, leaving the density unchanged. A constant density model would behave almost like the aimed fire model, so it will not be considered further herein.

\section{AREA FIRE PROPERTIES}

This section examines the behavior of the area fire salvo model and its combat implications. Appendix 1 contains the details of the mathematical derivations.

\subsection{The Outcome of One Salvo}

Begin by considering Red's attack against Blue. Red will cause no damage if its diluted offensive firepower is insufficient to overcome Blue's active defenses. That is, $\Delta B=0$ whenever $(\alpha A m B-z B) v \leq 0$. This can be simplified to show that Red inflicts no damage when $A \leq z /(\alpha m)$.

At the other extreme, Red can completely eliminate Blue with a single salvo if it can saturate its defenses and match its staying power. That is, $\Delta B=-B$ whenever $(\alpha A m B-z B) v \geq B$. Thus immediate elimination of Blue will occur whenever $A \geq(z+x) /(\alpha m)$.

These two expressions divide the potential size of Red's force into 3 ranges. When $A \leq$ $z /(\alpha m)$, Red is too weak to cause any damage. When $z /(\alpha m) \leq A<(z+x) /(\alpha m)$, then Red can damage Blue, but not immediately destroy it. Finally, when $(z+x) /(\alpha m) \leq A$, Red has enough firepower to eliminate Blue with a single salvo. 
If Blue has no active defense (i.e., $z=0$ ), then the first range disappears; Red always inflicts some damage. If staying power is negligible relative to the weapons used (i.e., as $x \rightarrow 0$ ), then the second range shrinks in size; after Red saturates the defenses, it quickly eliminates Blue.

The analysis above covers Red's attack, but equivalent results apply for Blue as well. Figure 1 illustrates how the two force sizes jointly determine the outcome of a salvo exchange. The 3 ranges for each side combine to produce 9 regions in total.

In the central region of this diagram, both sides lose part but not all of their force after one salvo, much as in the area fire Lanchester model. The diagonal dividing line represents cases where exactly the same proportion of starting force is lost by each side. The triangular area to the upper-left represents cases where Red $(A)$ loses proportionately more than Blue $(B)$, while the triangle to the lower-right indicates where Blue loses more than Red.

The bottom row of the diagram differs from the Lanchester model by representing cases where Red takes no damage; similarly, the left column shows Blue taking no damage. These overlap in the bottom-left to create a stalemate where neither side can hit the other. These regions are absent from the area fire Lanchester model, but exist in the aimed fire salvo model.

The top row represents scenarios where Red is eliminated after just one salvo, and the right column shows Blue being eliminated after one salvo. The top-right square therefore represents situations where both forces would be destroyed simultaneously. With area fire, such situations could only arise with unusually large quantities of firepower.

Note that it is the absolute size of each force (i.e., the numbers of units $A$ and $B$ ) that matters for Figure 1. In particular, the number of units deployed by one side determines the proportion of forces lost by the other side during that salvo. This is quite different from the aimed fire salvo model, where the relative size of the forces (i.e., ratio $B / A$ ) decides the outcome [3]. Interestingly, the size of a force here has no effect on its own proportion lost during one salvo, though it could help over the course of several salvos. Larger forces in fact suffer greater absolute losses per salvo, since the proportion lost remains constant.

Area fire also differs somewhat from aimed fire in regards to its lethality. Aimed fire salvo combat can be divided into 3 distinct categories (high, medium, and low lethality) based upon the qualities of the warships on each side, but independent of their quantities [3]. In the area fire case however, unit quantities influence the lethality level via the target densities. The two defining inequalities are shown below.

$$
m B n A \alpha \beta \geq(y+w)(z+x) \quad \text { and } \quad m B n A \alpha \beta \leq y z
$$

If Equation 5 is true, then the salvo exchange falls into the high lethality category, where at least one force is completely eliminated after a single salvo. Roughly speaking, battles have high lethality when diluted offensive fire is greater than the sum of defensive fire plus staying power. This is the same as in [3], except that the target densities $m B$ and $n A$ now appear as multipliers on the left side. Since both densities are less than one, their presence indicates that 
high lethality is more difficult to achieve with area fire, and becomes increasingly unlikely as the targeted area ratios decrease.

If instead Equation 6 is true, then the area fire salvo exchange falls into the low lethality category, where at least one force is unharmed. Battles have low lethality when diluted offensive fire is weaker than defensive power alone. In this case, the presence of the target densities indicates that low lethality is easier to achieve with area fire than with aimed fire, and becomes increasingly likely as targeted area ratios decrease. Roughly speaking, imprecise targeting information will tend to result in inconclusive battles.

If neither Equation 5 nor 6 is true, then the battle has a medium level of lethality.

\subsection{Performance Measures}

The Fractional Exchange Ratio (FER) summarizes a battle's outcome by comparing the proportions of units lost by the two sides, as in FER $=(\triangle A / A) /(\Delta B / B)$. Here, FER $>1$ indicates that Red is losing relatively more than Blue, while FER $<1$ indicates the reverse.

Of particular interest is FER $=1$, indicating that both sides lose the same proportion of forces. With area fire salvo combat, this parity occurs when $(\alpha m w A-z w)=(\beta n x B-y x)$. This is represented in Figure 1 by the diagonal dashed line in the center, which has slope $(\alpha w m) /(\beta x n)$. This slope provides a rough way to measure the value of one Red unit relative to one Blue opponent: multiply the offensive firepower, targeting precision, and staying power for each side, and then compare these two products. For each extra combatant that Red adds to its force before battle, Blue would need to add $(\alpha w m) /(\beta x n)$ more of its own to maintain parity.

The parity expression has (approximately) linear law behavior similar to Lanchester area fire, where the equivalent expression for parity would be simply $\alpha m A=\beta n B$ [12]. In both cases, an increase in the quantity of units on one side can be offset by a proportional improvement in the other side's quantity, quality, or targeting information. By contrast, aimed fire salvo combat exhibits (approximately) square law behavior [3]. These characteristics imply that the side with superior quality should prefer battles where both sides use area fire; it would therefore make extra efforts to conceal its location. Conversely, the more numerous side would do better with aimed fire, and thus should strive to precisely locate its opponent.

A similar intuition can be obtained by examining the continuous-time version of the area fire salvo model (as [3] briefly did for the aimed fire case) where the loss rates are differential equations. (This assumes that both sides can hit their opponents, so that both rates are non-zero.)

$$
d B / d t=-(\alpha A m B-z B) v \text { and } d A / d t=-(\beta B n A-y A) u
$$

These loss rates can be used to find a conserved quantity that relates them (see, e.g., [4, 12]); that is, a relationship that remains constant as the two forces are gradually destroyed.

$$
\text { constant }=(\alpha w m A-w z \ln (A))-(\beta x n B-x y \ln (B))
$$


This expression combines both linear and non-linear terms. The linear terms are the same as in the parity slope expression, and will dominate when force sizes are large. The natural logarithm terms induce some curvature, particularly when the force sizes are small.

With Lanchester models, the FER directly indicates who is winning the battle. But in the salvo context the definition of "winning" can depend on the ships' missions. Such missions could include sea denial, which requires eliminating the enemy; and survival, which requires that at least some friendly ships survive to carry out their mission [3].

The targeted area ratios influence how readily these missions can be accomplished. Smaller ratios shift the battle towards the lower-left on Figure 1. This makes it easier to survive but harder to achieve sea denial, because it is more difficult to inflict damage. Larger ratios shift the battle towards the upper-right on the diagram. It becomes easier to achieve sea denial, but harder to survive, because losses become heavier.

\subsection{Multiple Salvos}

Some battles could last for several salvos if neither side withdraws. The bottom-center and center-left regions of Figure 1 represent situations where the first salvo results in partial damage to one side but none to the other. Each subsequent salvo will again inflict losses only on that same side, whose strength will asymptotically approach zero, similar to what occurs in the Lanchester model [12]. Such a battle will remain in the same region of the diagram throughout its duration, absent any withdrawals or reinforcements.

The battle will progress differently, however, if it begins in the center region of the diagram. Here, the first salvo causes partial losses to both sides, as with the Lanchester case [12]. Unlike that case, however, these battles can shift into the bottom-center or the center-left region once one side becomes too weak to hit the other. Thereafter, only that side would suffer further casualties. These cases are represented in the magnified view of Figure 2 by the upperleft and lower-right corners; the arrows indicate the shift directions.

An even larger departure from both the Lanchester model and the aimed fire salvo model occurs if the battle begins in the upper-right corner of Figure 2; i.e., if the initial force strengths are close to parity and also close to causing immediate elimination. In this case, the first salvo exchange is damaging enough to push both sides simultaneously into the no-loss region, where both sides are unable to hit the other. Thus high lethality in the initial salvo can paradoxically lead to low lethality in subsequent ones.

More precisely, if Red's initial strength $A$ satisfies Equation 10 below, then Blue's survivors will be unable to inflict damage in future salvos. Likewise, if Blue's initial strength $B$ satisfies Equation 11, then Red's survivors will be unable inflict damage in future salvos.

$$
A \geq \frac{z+x}{\alpha m}-\frac{x y}{\alpha \beta m n B} \quad \text { and } \quad B \geq \frac{y+w}{\beta n}-\frac{w z}{\alpha \beta m n A}
$$


The effect of one salvo on the behavior of subsequent ones will therefore depend on which combination of these inequalities is satisfied.

(a) If both inequalities are true, then neither side will suffer further losses in later salvos;

(b) If the first is true but the second is false, then in later salvos Blue will no longer be able to damage Red, but Red will continue to damage Blue;

(c) If the first is false but the second is true, then in later salvos Red will no longer be able to damage Blue, but Blue will continue to damage Red; or,

(d) If both inequalities are false, then in the next salvo both sides will continue to suffer losses.

\section{THE HYBRID MODEL}

Some battles might be better represented by a model where one side uses area fire and the other uses aimed fire. For example, in Mahon's study of littoral combat [11], the army used aimed fire for their missiles while the navy used area fire for their guns. More generally, differences in the technologies and/or strategies of two opponents could easily lead to one possessing much better targeting information than the other.

Therefore, this section examines an aimed-fire-versus-area-fire hybrid model and compares it to the pure area fire model and the pure aimed fire model. For exposition purposes, Red is assumed to use area fire while Blue uses aimed fire. Thus Red losses will be calculated using Equation 2, and Blue losses using Equation 3.

For Blue's aimed fire, the key expressions are taken from [4] as follows. If $B / A \leq y / \beta$, Blue is too weak to cause damage. If $y / \beta \leq B / A<(y+w) / \beta$, then Blue hits Red's ships, but does not completely destroy them. Finally, if $(y+w) / \beta \leq B / A$, Blue eliminates Red with one salvo.

Figure 3 shows the possible salvo outcomes. The vertical boundaries that determine Blue losses are based upon the absolute size $A$ of Red, while the sloping boundaries that determine Red losses are based upon the relative size $B / A$ of Blue. The same 9 regions from Figure 1 appear again in Figure 3, but they are no longer rectangular.

The inequalities defining the high and low lethality levels, respectively, are shown below. Since only one of the targeted area ratios appears there, high lethality is more likely to occur with hybrid than with area fire, though it is less likely than with aimed fire. Similarly, low lethality is less likely to occur with hybrid fire than with area fire, but it is more likely than with aimed fire.

$$
m B \alpha \beta \geq(y+w)(z+x) \text { and } m B \alpha \beta \leq y z
$$

The expression for FER $=1$ in hybrid salvo combat also changes to become $m A \alpha v A-z v A$ $=\beta u B-y u A$, or equivalently $(m \alpha w) A^{2}+(y x-z w) A-(\beta u B)=0$. This quadratic is represented in the center of Figure 3 by the dashed curve. In situations where both sides make comparable use of active and passive defenses (so that $y x=z w$ ), the condition for FER $=1$ simplifies to $(m \alpha w) A^{2}$ $=(\beta x) B$. This is structurally similar to the conserved quantity expression from the Lanchester hybrid model [4], where $\left((m \alpha) A^{2}-(2 \beta) B\right)=$ constant. These expressions imply several things. 
First, they show that the hybrid model possesses a mix of (approximately) linear and square law properties. If the area fire side improves in quality (i.e., offensive firepower or targeting precision), then the aimed fire side can match that with a proportional increase in either the quality or the quantity of its force, as in the linear law case. However, if the area fire side increases in quantity, then to maintain parity the aimed fire side would need to respond with a squared increase in its own quantity or quality, similar to the square law case. This also makes the concept of parity more complex here, as the relative "worth" of the combat units depends on the numbers present.

Second, the fact that the targeted area ratio appears on the left side of the equation but not the right represents the advantage of using aimed fire. This information advantage [10] could help a force to compensate for being outclassed and/or outnumbered, as [11] suggested. However, because the targeted area ratio appears on the term that is squared (i.e., it multiplies $A^{2}$ ), that information advantage diminishes as force sizes and target densities increase.

Another way to evaluate this advantage is to compare the FERs for aimed and hybrid fire. Taking their ratio and simplifying leads to the following expression. It is relevant where both sides can hit each other, so that the numerator and denominator are both strictly positive.

$$
F E R_{\text {aimed }} / F E R_{\text {hybrid }}=(m B \alpha A-z B) /(\alpha A-z B)
$$

This ratio is always less than one, since the target density $0<m B<1$, and it indicates how much worse Red performs due to using area fire instead of aimed fire. The performance gap increases when targeting information is poor (i.e., as $m B \rightarrow 0$ ), and/or when forces are closely matched in overall defensive and offensive strength (i.e., as $z B \rightarrow \alpha A$ ).

With regards to multiple salvos, it is again possible for a battle to start in the middle region of Figure 3 where both sides take damage, but then shift into a region where one or both sides suffer no further losses. The concept is the same as before, but the equations for the hybrid model are as follows.

$$
A \geq \frac{B \beta(w x+w z+x y)}{B \beta \alpha m w+x y(y+w)} \quad \text { and } \quad \frac{B}{A} \geq \frac{y+w}{\beta}-\frac{w z}{\beta \alpha m A}
$$

\section{CONCENTRATION OF FIRE}

A force using area fire could face serious problems if it spreads its firepower across a large area. The number of rounds heading towards any given target could become so small that defensive fire intercepts them all, i.e., if $\alpha A m \leq z$. One way to mitigate this dilution would be to focus the attack on just a portion of the original target area. For example, instead of Red firing into all $1 / m$ subsections of the target area, it could choose some smaller number $t$. Red would choose $t$ so that the quantity of missiles fired at each subsection is large enough to overcome a target's defenses, if one happens to be there. (Any targets outside the chosen portion would remain unharmed.) This approach can be formalized by adapting results from [1] as follows. 
Let $S$ stand for the total number of offensive missiles in Red's salvo. Define salvo size $S^{* *} \equiv(z+x)$ to represent the number of missiles required to destroy one target, and $S^{*} \equiv(z+$ $x / 2$ ) as the number required to destroy half of one target. Further define a set of salvo size "breakpoints" $S_{t, t+1} \equiv(z+t(z+x))=S_{t-1, t}+S^{* *}, t \in\{0,1,2, \ldots\}$. Then the following properties hold for the area fire salvo model.

(a) For any given number of potential target locations $t$, a total salvo size of $S=t S^{* *}$ is "optimal" in the sense that it is the smallest quantity that will immediately eliminate all targets that might be present. If $t=1 / \mathrm{m}$, then a salvo of size $S=S^{* *} / \mathrm{m}$ would be needed;

(b) For a given number of target locations $t$, any salvo size $S$ in the range $S_{t-1, t} \leq S \leq S_{t, t+1}$ will inflict average losses at least as large against $t$ locations as against $t-1$ or $t+1$ locations;

(c) To maximize the average losses inflicted with a given salvo size $S$, the optimal number of locations to attack is the smallest integer value of $t$ such that $t \geq(S-z) /(z+x)$;

(d) Under these rules, the average number of missiles per targeted location will always be at least $S^{*}$ (i.e., $S / t \geq S^{*}$ ), and it will converge to $S^{* *}$ for large $S$ and $t$ (i.e., $S / t \rightarrow S^{* *}$ as $S \& t \rightarrow \infty$ ).

Case (a) indicates the number of missiles needed by Red to achieve the ideal of eliminating Blue. Case (b) suggests the preferred number of missiles to use, assuming that Red has already chosen the number of locations at which to fire. Most usefully, case (c) indicates the number of locations to target, given the limited number of missiles actually available.

\section{DISCUSSION}

\subsection{Concealing and Uncovering Information}

The properties of the area and hybrid fire salvo models provide a reminder of the value of information in combat; see e.g., $[4,10,11]$. Each side wants precise targeting information about their enemy, while denying them the same; e.g., Red prefers $m \rightarrow 1$ but $n \rightarrow 0$. The analysis herein suggests that a force with weaker but more numerous units could do better in aimed fire battles than in area fire ones, as the former's square law properties favor quantity over quality. Thus they should seek to accurately locate their opponents, even if at the risk of revealing their own positions.

With area fire, the precision of location information is represented by the targeted area ratios $m$ and $n$. Attackers can improve these ratios in several ways. They can obtain better tactical intelligence so as to more precisely locate their targets before opening fire, and employ "smarter" weapon guidance systems that can locate their own targets once they reach the enemy's vicinity. Conversely, defenders can decrease the targeted area ratios by obscuring their locations; this can involve active measures such as chaff and decoys [9], or passive measures such as radio silence and stealth technology [6]. For example, if stealth warship designs make it more difficult for ASCMs to acquire targets, then smaller targeted area ratios could represent the use of such technology.

\subsection{Limitations and Future Work}


This paper builds on the numerical work in [11] by providing an analytical study of the area fire and hybrid fire versions of the salvo combat model. However, both studies deal only with deterministic models, whereas actual combat is full of random variation. Thus future research could extend area fire to the stochastic version of the salvo model [2]. A stochastic treatment would be particularly appropriate when target densities are low, as then the probability of hitting any given target becomes small.

There are several potential applications of the hybrid fire model aside from those already mentioned. For example, it might be used to represent certain kinds of antisubmarine warfare. A submarine may know the exact location of enemy warships when it launches its attack, while those warships know only the approximate location of the submarine. A salvo model will become particularly appropriate if hard-kill defenses, such as anti-torpedo torpedoes [13], are ever successfully deployed.

The model might also be adapted to represent some kinds of land battles. For example, in fall 2012, Gaza strip militants fired over 1500 unguided rockets in area fire attacks against civilian targets in Israel. Israel reportedly intercepted 421 of these using its Iron Dome missile defense systems [5]. If a future attack involves the militants launching their rockets in coordinated salvos (instead of sporadic streams) and directing them against the Israeli defense systems (instead of civilian targets), then a salvo-style model could become appropriate.

Future research could also incorporate more tactical complexity into the situations being modeled. For example, naval forces employ both short-range point-defense systems and longrange area-defense systems. Analyzing layered defenses like these would be more difficult, but could lead to some interesting results.

\section{ACKNOWLEDGEMENTS}

Thanks are due to Professor Jeffery Kline for his comments regarding potential applications of area fire in surface warfare. Thanks are also due to two anonymous reviewers and the associate editor for their suggestions.

\section{REFERENCES}

[1] MJ Armstrong. Effective attacks in the salvo combat model: salvo sizes and quantities of targets. Naval Research Logistics 54 (1), 2007, 66-77.

[2] MJ Armstrong. A stochastic salvo model for naval surface combat. Operations Research 53 (5), 2005, 830-841.

[3] MJ Armstrong. Effects of lethality on naval combat models. Naval Research Logistics 51 (1), 2004, 28-43.

[4] SJ Deitchman. A Lanchester model of guerrilla warfare. Operations Research 10 (6), 1962, 818-827.

[5] TT Ha. Israeli missile defense built with eye to the future. Globe and Mail, 24 Nov 2012.

[6] RC Harney. Broadening the trade space in designing for warship survivability. Naval Engineers Journal 122 (1), 2010, 49-63. 
[7] WP Hughes. A salvo model of warships in missile combat used to evaluate their staying power. Naval Research Logistics 42 (2), 1995, 267-289.

[8] E Kaplan, M Kress, R Szechtman. Confronting entrenched insurgents. Operations Research 58 (2), 2010, 329-341.

[9] J Kline. Exploring the effects of counter targeting in naval warfare. Working paper, $1 \mathrm{Jul}$ 2008, Operations Research Department, Naval Postgraduate School, Monterey, CA.

[10] TW Lucas, JE McGunnigle. When is model complexity too much? Illustrating the benefits of simple models with Hughes' salvo equations, Naval Research Logistics 50 (3), 2003, 197-217.

[11] CM Mahon. A Littoral Combat Model for Land-Sea Missile Engagements. Master's thesis, Sep 2007, Operations Research Department, Naval Postgraduate School, Monterey, CA.

[12] JG Taylor. Lanchester Models of Warfare. 1983, Operations Research Society of America.

[13] USNI. Navy develops torpedo killing torpedo. News report, United States Naval Institute. 20 Jun 2013. http://news.usni.org/2013/06/20/navy-develops-torpedo-killing-torpedo

[14] L Xinqui, T Shoulin. Damage effectiveness analysis of submunition against aircraft carrier 1. Zhanshu Daodan Jishu, 1 Nov 2006.

\section{APPENDIX 1. MATHEMATICAL DERIVATIONS.}

Note: the following derivations often use the substitutions $w=1 / u$ and $x=1 / v$.

\section{Key force levels for 4.1.}

If $(\alpha A m B-z B) v \leq 0$, then $\alpha A m-z \leq 0$ and so $A \leq z /(\alpha m)$.

If $(\alpha A m B-z B) v \geq B$, then $\alpha A m v-z v \geq 1, \alpha A m-z \geq x$, and so $A \geq(z+w) /(\alpha m)$.

High lethality exists if at least one side is eliminated in one salvo exchange regardless of the $B / A$ force ratio. Blue is eliminated in one salvo if $A \geq(z+x) /(\alpha m)$ or equivalently $B / A \leq$ $(\alpha m B) /(z+x)$. Red is eliminated in one salvo if $B \geq(y+w) /(\beta n)$ or equivalently $B / A \geq$ $(y+w) /(\beta n A)$. Thus, for any value of ratio $B / A$, at least one of those expressions will always be true if $(y+w) /(\beta n A) \leq(\alpha m B) /(z+x)$, which is equivalent to $(y+w)(z+x) \leq \alpha \beta(m B)(n A)$.

Low lethality exists if at least one side is unable to strike the other regardless of the $B / A$ force ratio. Red suffers no loss if $B \leq(y) /(\beta n)$ or $B / A \leq(y) /(\beta n A)$. Blue suffers no loss if $A \leq$ $(z) /(\alpha m)$ or $B / A \geq(\alpha m B) /(z)$. Thus at least one of those expressions will always be true if $(y) /(\beta n A) \geq(\alpha m B) /(z)$, or equivalently $y z \geq \alpha \beta(m B)(n A)$.

\section{Loss ratios for 4.2.}

For the FER, begin by simplifying the definition and setting it equal to 1 .

$$
F E R=\frac{\Delta A / A}{\Delta B / B}=\frac{(\beta B n A-y A) u / A}{(\alpha A m B-z B) v / B}=\frac{(\beta B n-y) u}{(\alpha A m-z) v}=1
$$


Rearrange this to get $\alpha m v A-z v=\beta n u B-y u$, then multiply by $w x$ to get $\alpha m w A-z w=\beta n x B-y x$. The slope of the diagonal parity line follows from the behavior of this expression. As $A$ increases, Blue must increase $B$ by a factor of $(\alpha m w) /(\beta n x)$ to maintain $\alpha m w A=\beta n x B$.

For the continuous time version, begin with the loss rate differential equations. Combine them via the chain rule to get $d A / d B$.

$$
d A / d B=(d A / d t) /(d t / d B)=(u \beta B n A-u y A) /(v \alpha A m B-v z B)
$$

Rearrange terms, divide by $A B$, and integrate to get the conserved quantity expression.

$$
\begin{aligned}
& (v \alpha A m B-v z B) d A=(u \beta B n A-u y A) d B \\
& \left(v \alpha m-v z A^{-1}\right) d A=\left(u \beta n-u y B^{-1}\right) d B \\
& v \alpha m A-v z \ln (A)=u \beta n B-u y \ln (B)+\text { constant }
\end{aligned}
$$

\section{Salvo transitions for 4.3 .}

To derive the first inequality, suppose that Red's initial salvo weakens Blue enough that it can cause no further damage; i.e., $(B+\Delta B) \leq y /(\beta n)$ and so $(B-(\alpha A m B-z B) v) \leq y /(\beta n)$. Then:

$$
\begin{aligned}
& x-(\alpha A m-z) \leq y x /(\beta n B) \\
& x+z-(y x) /(\beta n B) \leq \alpha m A \\
& (x+z) /(\alpha m)-(y x) /(\alpha m \beta n B) \leq A
\end{aligned}
$$

A similar derivation finds the limit for Blue's initial strength $B$.

When both inequalities are true, after one salvo both sides will be too weak to inflict further damage, as in (a). If only one is true, then that side will be able to cause further damage, but its opponent will not, as in (b) \& (c). If neither is true, then both sides can continue causing damage, as in (d). Note that all 4 cases may not exist for any given set of parameters.

\section{Hybrid model for 5 .}

The inequalities that define the lethality levels are derived in the same manner as in section 4.1 above, except that the term $n A$ is deleted because Blue is using aimed fire.

Regarding the FER, simplify the definition and set it equal to 1.

$$
F E R=\frac{\Delta A / A}{\Delta B / B}=\frac{(\beta B-y A) u / A}{(\alpha A m B-z B) v / B}=\frac{(\beta B-y A) u}{(\alpha A m-z) v A}=1
$$

Rearrange to get $\alpha m v A^{2}-z v A=\beta u B-y u A$ and multiply by $w x$ to get $\alpha m w A^{2}-z w A=\beta x B-y x A$. If $z / x=y / w$, then $z w=y x$; and so $\alpha m w A^{2}=\beta x B$, or equivalently $\alpha m y A^{2}=\beta z B$.

This can be compared to the FER for aimed fire by taking their ratio and simplifying. 


$$
\frac{F E R_{\text {aimed }}}{F E R_{\text {hybrid }}}=\frac{(\beta B-y A) u / A}{(\alpha A-z B) v / B} \frac{(\alpha A m B-z B) v / B}{(\beta B-y A) u / A}=\frac{(\alpha A m B-z B)}{(\alpha A-z B)}
$$

For transitions after one salvo, first suppose that Blue's aimed fire weakens Red so that it can no longer cause damage, $(A+\Delta A) \leq z /(\alpha m)$, and so $A-(\beta B-y A) u \leq z /(\alpha m)$. Then:

$$
\begin{aligned}
& w A+y A-\beta B \leq(z w) /(\alpha m) \\
& (w+y) A-(z w) /(\alpha m) \leq \beta B \\
& (w+y) /(\beta)-(z w) /(\alpha m \beta A) \leq B / A
\end{aligned}
$$

Next suppose that Red's area fire weakens Blue so that it can no longer cause damage, as in $(B+\Delta B) /(A+\Delta A) \leq y / \beta$. Then proceed as follows.

$$
\begin{aligned}
& \frac{B-(\alpha A m B-z B) v}{A-(\beta B-y A) u} \leq \frac{y}{\beta} \\
& \beta B+\beta z v B-\beta \alpha A m v B \leq y A+y^{2} u A-y u \beta B \\
& \beta B+z v \beta B+y u \beta B \leq y A+y^{2} u A+v \alpha A m \beta B \\
& \frac{\beta B+z v \beta B+y u \beta B}{y+y^{2} u+v \alpha m \beta B}=\frac{w x \beta B+w z \beta B+x y \beta B}{y w x+y^{2} x+w \alpha m \beta B} \leq A
\end{aligned}
$$

\section{Concentration of fire for 6.}

For part (b), first decide whether to engage 0 or 1 location. To saturate a target's defense requires $S_{0,1}=z$ missiles, in which case the loss is the same (i.e., none) whether firing at 1 or 0 . Once the salvo exceeds $S_{0,1}$ it becomes better to fire at 1 location (i.e., 1 potential target).

Next decide whether to engage $t$ or $t+1$ target locations. A salvo size of $S_{t, t+1}=\{z+t(z+$ $x)\}$ would be indifferent between $t$ or $t+1$ targets, as $z$ missiles are wasted either way. But as soon as $S$ exceeds $S_{t, t+1}$ it becomes better to shoot at $t+1$ locations.

Part (c) is derived by re-arranging the relation in part (b) to solve for $t$.

For the first part of (d), divide $S_{t-1, t}$ from part (b) by $t$ and proceed as follows.

$$
S_{t-1, t} / t=\{z+(t-1)(z+x)\} / t=\{z+(x)(t-1) / t\} \geq\{z+x / 2\}=S^{*}
$$

The second part is obtained by taking the limit of $S_{t-1, t} / t$ as $t \rightarrow \infty$.

$$
\lim _{t \rightarrow \infty}\{(z+(t-1)(z+w)) / t\}=(z+w)-\lim _{t \rightarrow \infty}(w / t)=S * *
$$

\section{APPENDIX 2. NUMERICAL EXAMPLE.}

Note: the parameter values used below were chosen to highlight the properties of the equations. They are not necessarily representative of any particular battle situation. 
Let Red have offensive firepower $\alpha=6$ missiles per unit, defensive firepower $y=2$ interceptions per unit, and staying power $w=2$ hits per unit, so $u=0.5$ units lost per hit. Suppose Blue has weaker units, with offense $\beta=4$, defense $z=1$, and staying power $x=1$, so $v=$ 1. Let the targeted area ratios be $m=n=0.05$, so that the areas being fired at are $1 / 0.05=20$ times larger than the area covered by each missile.

\section{Area Fire}

Suppose that both sides use area fire. If each has $A=B=4$ units, then during the salvo Blue will lose $(4 \times 6 \times 0.05 \times 4-1 \times 4) / 1=0.8$ units and Red will lose none. This is a low lethality situation when only 4 units are on each side, as $(0.05 \times 4 \times 0.05 \times 4 \times 6 \times 4) \leq(2 \times 1)$. Any Red force with $A \leq 1 /(6 \times 0.05)=3.33$ units will be unable to damage Blue. Red would need $A \geq$ $(1+1) /(6 \times 0.05)=6.67$ units to eliminate Blue with a single salvo.

Parity occurs when $(6 \times 0.05 \times 1 A-1 \times 1)=(4 \times 0.05 \times 0.5 B-2 \times 0.5)$, which simplifies to $3 A=$ $B$. Each side loses the same proportion of its force when Blue has 3 times as many units as Red. In that sense, 1 Red unit is "worth" 3 Blue units. By contrast, if both sides were using aimed fire [3], then parity would occur when $3^{1 / 2} A=B$, and each Red would be worth 1.73 Blue.

To see how battles could evolve over several salvos, consider the following cases.

- If Blue starts with 15 units and Red with 5, then after 1 salvo they will have 7.50 and 2.50 survivors respectively, with neither side being able to hit the other any further.

- If Blue starts with 13 while Red has 5, then after 1 salvo they will have 6.50 and 3.50 left respectively; in later salvos, Red will suffer no loss while gradually destroying Blue.

- If Blue starts with 15 while Red has 4, then after 1 salvo they will have 12.00 and 2.00 respectively; in later salvos, Blue will suffer no loss while gradually destroying Red.

\section{Hybrid Fire}

If Red uses area fire while Blue uses aimed fire, then FER $=1$ will occur whenever $A^{2}=$ $B(4 \times 1) /(6 \times 2 \times 0.05)=6.67 B$. If Red has 4 units, Blue will need $B=4^{2} / 6.67=2.40$ to obtain parity; each side would lose $20 \%$ of its force. If Red instead has 5 units (a $25 \%$ increase), then Blue would need 3.75 (a 56\% increase); each side would lose $50 \%$ of its force. If each side has 5 units, then the hybrid-to-aimed FER ratio is $(0.05 \times 5 \times 6 \times 5-1 \times 5) /(6 \times 5-1 \times 5)=7.5 / 25=30 \%$. Red only performs $30 \%$ as well when it is forced to use area fire.

\section{Effective attacks}

Assume that Blue, using area fire, focuses its firepower on a limited area. Against the same Red unit type as before, $S^{* *}=(2+2)=4$ missiles, $S^{*}=(2+2 / 2)=3$ missiles, and $S_{t, t+1}=$ $2+4 t$. If Red's units lie within an area of $t=1 / 0.05=20$ possible locations, then ideally Blue would fire $20 \times 4=80$ missiles to completely eliminate Red. If Blue has only 16 missiles available, then to inflict the largest average loss they should direct those missiles at just (16$2) /(2+2)=3.5 \approx 4$ of the possible locations. Any salvo size between $S_{3,4}=14$ missiles and $S_{4,5}=$ 18 missiles would do at least as much damage to 4 target locations as against 3 or 5 . 
Table 1. List of symbols used.

\begin{tabular}{lcc}
\hline & Red & Blue \\
\hline Number of combat units & $A$ & $B$ \\
Offensive firepower per unit & $\alpha$ & $\beta$ \\
Defensive firepower per unit & $y$ & $z$ \\
Staying power per unit & $w$ & $x$ \\
Loss suffered by defender per hit & $u$ & $v$ \\
Targeted area ratio for attacker & $m$ & $n$ \\
\hline
\end{tabular}

Figure 1. Possible outcomes after 1 salvo with area fire by both sides.

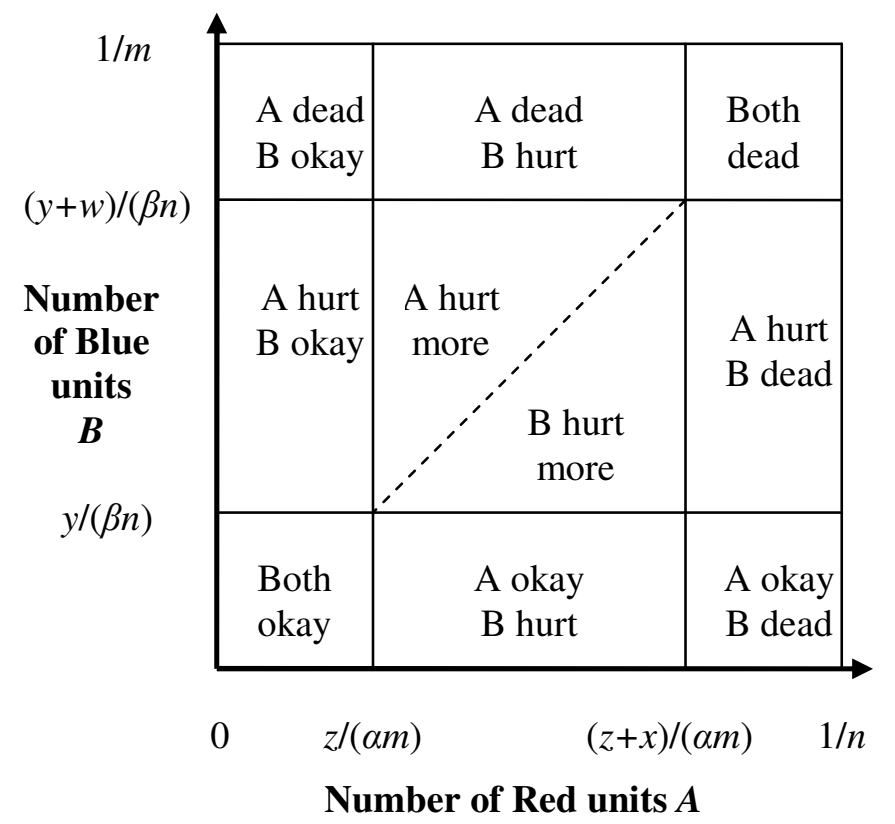


Figure 2. Transitions out of the center region after a salvo.

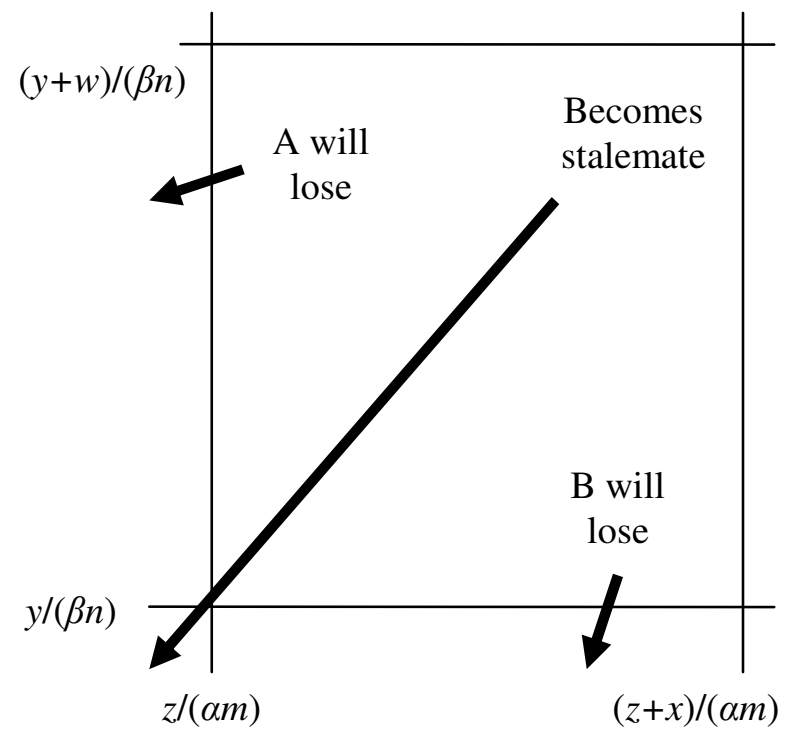

Figure 3. Possible outcomes after 1 salvo where Red uses area fire and Blue uses aimed fire.

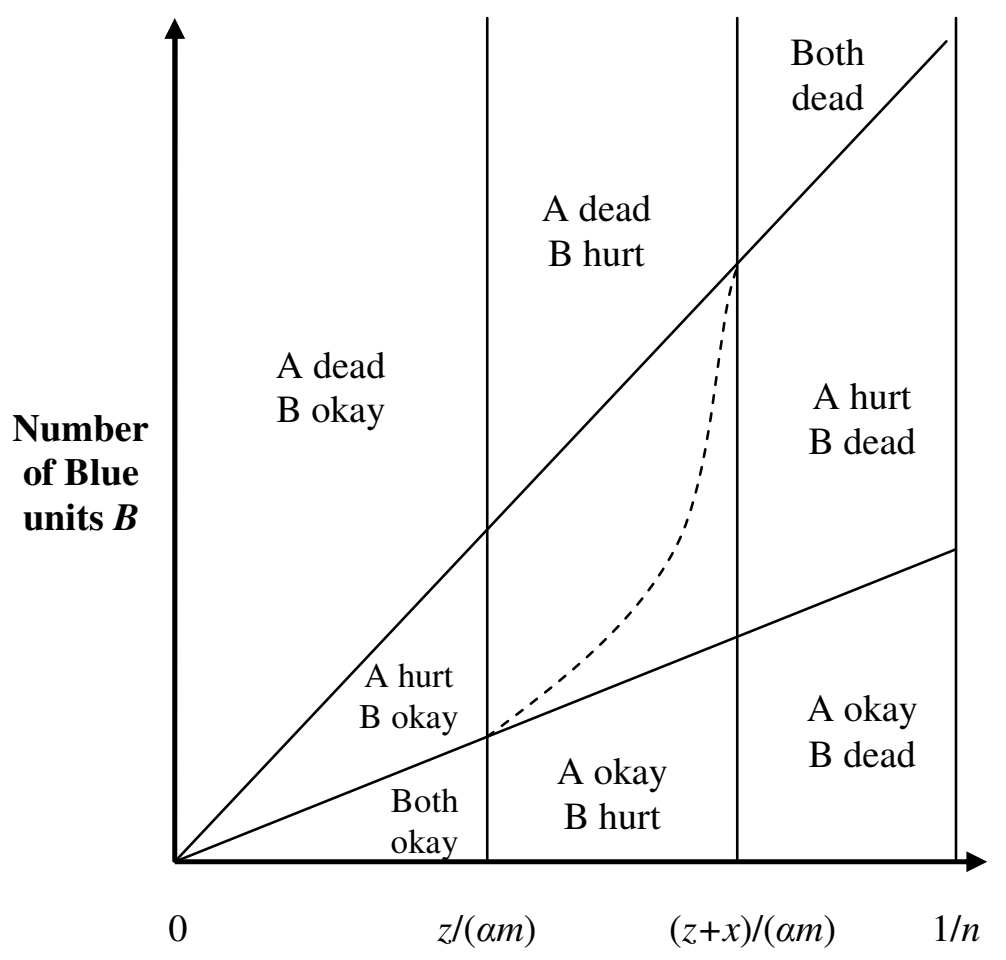

Number of Red units $A$ 